\title{
CME. Hypertonie in der Schwangerschaft
}

Eggersmann, Tanja K ; Zechmann, Stefan ; Zimmerli, Lukas

DOI: https://doi.org/10.1024/1661-8157/a002035

Other titles: CME. Hypertension in pregnancy

Posted at the Zurich Open Repository and Archive, University of Zurich ZORA URL: https://doi.org/10.5167/uzh-112556

Journal Article

Originally published at:

Eggersmann, Tanja K; Zechmann, Stefan; Zimmerli, Lukas (2015). CME. Hypertonie in der Schwangerschaft. Praxis, 104(12):605-614.

DOI: https://doi.org/10.1024/1661-8157/a002035 
Titel: Hypertonie in der Schwangerschaft

Text (max. 3300 Zeichen mit Leerzeichen)

Hypertonie in der Schwangerschaft ist eine medizinische Risikokonstellation für Mutter und Kind, der es in der Praxis aufgrund von Anpassung im Therapie- und Überwachungsregime besondere Aufmerksamkeit zu schenken gilt.

6-8\% aller Schwangeren haben bereits eine vorbestehende arterielle Hypertonie (HTN) und die Inzidenz hierfür ist weiter zunehmend. Die HTN in der Schwangerschaft ist definiert als gemessener Blutdruckwert (BD-Wert) von mindestens $140 \mathrm{mmHg}$ systolisch und/oder $90 \mathrm{mmHg}$ diastolisch und wird je nach zeitlichem Auftreten in eine schwangerschaftsunabhängige oder -bedingte HTN eingeteilt (siehe Tab. 1, 2).

Beim Auftreten präkonzeptionell oder in der ersten Schwangerschaftshälfte $(<20$

Schwangerschaftswochen, SSW) zählt die HTN zur chronischen Form und ist mit einem Fortbestehen $>6-12$ Wochen postpartum ( $p p$ ) vergesellschaftet. Dabei tritt gehäuft HTN Grad 1 auf, welche bei guter Nierenfunktion eine gute Prognose hat.

Tritt die HTN nach der vollendeten 20. SSW auf, ist diese per definitionem schwangerschafts-bedingt und bildet sich meist innerhalb 6 (-12) Wochen pp zurück. Die Ursache dieser transienten HTN (Gestationshypertonie) ist nicht endgültig gesichert.

Zeigen sich erhöhte BD-Werte in Kombination mit einer signifikanten

Eiweissausscheidung im Urin der Patientin (Proteinurie $>300 \mathrm{mg} / 24 \mathrm{~h}$ ), handelt es sich um das Bild der Präeklampsie. Die schwerste Ausprägung der Präeklampsie wird Eklampsie genannt und stellt einen lebensbedrohlichen Zustand für Mutter und Kind dar [1, 2].

Tabelle 1: Schweregrad der Hypertonie nach Höhe des Blutdruckes (in mmHg) [3]

\begin{tabular}{|l|l|l|}
\hline Kategorie & Systolisch & Diastolisch \\
\hline Grad 1 Hypertonie & $140-159$ & $90-99$ \\
\hline Grad 2 Hypertonie & $160-179$ & $100-109$ \\
\hline Grad 3 Hypertonie & $\geq 180$ & $\geq 110$ \\
\hline
\end{tabular}


Tabelle 2: Bluthochdruckformen in der Schwangerschaft

\begin{tabular}{|l|l|l|}
\hline & schwangerschaftsunabhängig & schwangerschaftsbedingt \\
\hline $\begin{array}{l}\text { Auftreten } \\
\text { anhaltende } \\
\text { Hypertonie }\end{array}$ & $>20$ SSW & $>20$ SSW \\
\hline $\begin{array}{l}\text { ohne Proteinurie } \\
\text { mit Proteinurie } \\
(>\mathbf{3 0 0 m g / 2 4 h )}\end{array}$ & $\rightarrow$ Arterielle Hypertonie & $\rightarrow$ Gestationshypertonie \\
\hline
\end{tabular}

Generell gilt es bei der Einstellung des BD während der Schwangerschaft zurückhaltend vorzugehen, da eine aggressive Senkung zu einer fetalen Wachstumsrestriktion führen kann [4]. Bislang ist einzig eine mütterliche Indikation zur Vermeidung von zerebralen und kardiovaskulären Komplikationen gesichert. Ein Nutzen für die fetale Entwicklung oder eine Verbesserung der kindlichen Prognose konnte bislang nicht nachgewiesen werden [5].

Im Idealfall sollte die Betreuung und Behandlung der Patientinnen bereits vor der Schwangerschaft stattfinden. Dabei sollte über die teratogene Wirkung einiger antihypertensiver Medikamente und die Notwendigkeit der medikamentösen Umstellung im Falle einer Schwangerschaft aufgeklärt werden. Erste Lifestyle-Änderungen sollten bereits zu diesem Zeitpunkt stattfinden. Eine Gewichtsreduktion bei Adipositas durch ausgewogene Ernährung und regelmässige körperliche Aktivität führt dabei nicht nur zur besseren Einstellung des BD, sondern reduziert auch das Risiko eine Präeklampsie zu entwickeln [6]. Daher ist ein Normalgewicht bereits vor der Schwangerschaft anzustreben [7]. Die Schwangere sollte ausserdem über mögliche Anzeichen von Präeklampsie, Eklampsie oder des HELLP-Syndroms informiert werden, um eine rechtzeitige Behandlung einleiten zu können.(3285)

\section{Im Artikel verwendete Abkürzungen}

HTN: arterielle Hypertonie

BD: Blutdruck

24h-BDM: 24 Stunden Blutdruckmessung

pp: post partum

SSW: Schwangerschaftswoche 
Klinik und Komplikation

Maximal 3000 Zeichen inkl. Leerzeichen, kommen Tabellen oder Abb. hinzu entsprechend weniger.

Klinik

Das Vorliegen einer HTN bleibt bei fehlendem Screening häufig lange unerkannt und wird erst durch seine Komplikationen klinisch auffällig.

\section{Komplikationen}

Bei schwangeren Frauen mit chronischer HTN zeigte sich ein signifikant erhöhtes Risiko von Plazentalösung, fetaler Wachstumsrestriktion sowie Frühgeburtlichkeit. Zusätzlich haben sie im Vergleich zu anderen Schwangeren ein um das fünffach erhöhtes Risiko eine Präeklampsie zu entwickeln (17-25\% vs. 3-5\%), wobei der Schweregrad der Präeklampsie mit der Dauer der bestehenden HTN korreliert [2, 8].

Präeklampsie ist eine nach der 20. SSW auftretende schwangerschafts-spezifische Multiorganerkrankung mit mütterlicher und fetaler Manifestation, die in der Regel nach Beendigung der Schwangerschaft abklingt. Sie ist mit einer fünffach gesteigerten perinatalen Mortalität vergesellschaftet und ist weltweit jährlich für mindestens 50000 mütterliche Todesfälle verantwortlich. Maternale renale und zerebrovaskuläre Dysfunktion mit Endorganschaden und fetale Wachstumsretardierung prägen das klinische Erscheinungsbild [9].

Tabelle 3: Risikofaktoren für Entwicklung einer Präeklampsie und Eklampsie [9]

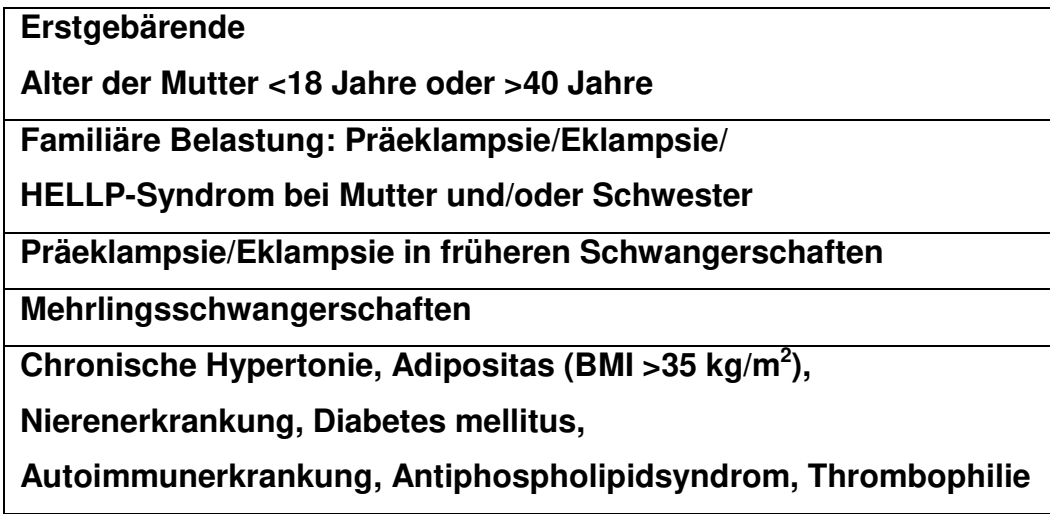

Die schwere Form der Präeklampsie mit klonisch-tonischem Krampfanfall und Bewusstlosigkeit wird Eklampsie genannt. Entwickelt die Patientin die laborchemische Trias aus Hämolyse, erhöhten Leberenzymen und erniedrigten Thrombozyten, spricht man per definitionem von einem HELLP Syndrom. Gemeinsam ist diesen genannten 
Komplikationen, dass nicht zwingend eine HTN vorrausgegangen sein muss. Da derzeit keine kausale Therapie zur Verfügung steht, besteht die rein supportive Behandlung aus intensivmedizinischer Betreuung und endet häufig in der Entbindung [10].

\section{Gefahr in der Praxis rechtzeitig erkennen und richtig Handeln}

Merke: Die ambulante Betreuung und Überwachung einer Patientin mit HTN in der Schwangerschaft ist nur bei Grad 1 einer schwangerschaftsunabhängigen HTN und ausgeschlossenen Risiken für Mutter und Kind möglich. Dabei muss eine adäquate Compliance der Schwangeren und die Gewährleistung von wöchentlichen ärztlichen Kontrollen sowie häuslichen BD-Messungen sichergestellt sein. Alle anderen Formen benötigen wenigstens die Überweisung in ein Zentrum und gegebenenfalls eine stationäre Überwachung oder eine intensivmedizinische Betreuung. (2678) 
Abklärungsstrategie

Maximal 3000 Zeichen inkl. Leerzeichen, kommen Tabellen oder Abb. hinzu entsprechend weniger.

Neben einer internistischen sowie schwangerschafts-spezifischen Anamnese und einer klinischen Untersuchung sind bei diesem Patientenkollektiv auch spezifische laborchemische Abklärungen notwendig.

Blutdruckmessung bei Schwangeren:

1. Die BD-Messung sollte an der sitzenden Patientin erfolgen, da der BD in Rückenlage (z.B. durch das Vena-Cava-Kompressionssyndrom) unterschätzt werden kann.

2. Der systolische BD-Wert wird durch das Hörbarwerden der Korotkoffgeräusche, der diastolische durch das Aufhören derselben bestimmt. Bei ca. 15\% der Schwangeren entsteht durch physiologische Hyperzirkulation im Verlauf der Schwangerschaft ein sogenanntes Nullphänomen, welches dazu führt, dass die Geräusche bis zum Wert 0 mmHg gehört werden können. In diesem Fall markiert ein deutliches Leiser- und Tieferwerden der Geräusche den diastolischen BD-Wert [11].

3. Primär sollte die Messung an beiden Armen und später - bei geringen Differenzen - am Arm mit den höher gemessenen Werten erfolgen. Neben einer adäquaten, an den Oberarmumfang adaptierten Manschettengrösse, sollte ein für Schwangere validiertes BD-Messgerät verwendet werden [12].

4. Erst mehrere wiederholt erhöhte BD-Messungen in Abstand von mindestens 4-6 Stunden in entspannter Atmosphäre können Hinweis auf das Vorliegen einer HTN geben.

5. Die ambulante 24-h-BDM ist eine empfohlene Methode, um eine HTN differentialdiagnostisch weiter abzuklären (z.B. Ausschluss einer Weisskittel-HTN). Ausserdem ermöglicht sie die Diagnose einer abendlichen/nächtlichen Hypertonie. Eine Umkehr des zirkadianen BD-Rhythmus in der Schwangerschaft steht in Zusammenhang mit dem Auftreten einer Präeklampsie und wird durch Praxis-BD Messungen oft übersehen [13, 14].

6. Zu beachten gilt, dass es im ersten Trimenon physiologischerweise bei gleichzeitigem Anstieg des Herzminutenvolumen, zu einem Abfall des systemischen Gefässwiderstandes und des mittleren BD-Wertes kommt. Gegen Ende der Schwangerschaft werden häufig wieder hypertone BD-Werte gemessen, die den präkonzeptionellen Werten ähneln [15-17]. 


\section{Urinkontrollen zum Ausschluss einer Präeklampsie}

Goldstandard zur Detektion einer Proteinurie ist die Messung der Proteinmenge im 24Stunden-Urin (Definitionen siehe Kasten). Aufgrund der schwierigen Umsetzung im ambulanten Setting etabliert sich zunehmend der Urin-Schnelltest (Urinstix) in Verbindung mit der Berechnung des Albumin-Kreatinin-

Quotienten aus dem Spontanurin als Screening-Methode. Dabei ist zu beachten, dass bereits der Nachweis einer Spur (+1) Eiweiss als abklärungsbedürftig anzusehen ist und weitere Untersuchungen notwendig macht.

\section{Entscheidende Laborparameter}

a) Basislabor: u.a. Blutstatus, Transaminasen, Elektrolyte, Kreatinin, sowie Urinstix und die Berechnung des Albumin-Kreatinin-Quotienten im Urin.

b) Erweitertes Labor: Fragmentozyten, freies $\mathrm{Hb}$ im Serum, LDH, Haptoglobin, Harnsäure, Quick, PTT, ATIII, Fibrinogen, D-Dimer, PIGF, sFIt-1/PIGF-Quotient (2922)

Diagnosekriterien/Ursachen/Differenzialdiagnose

Maximal 3000 Zeichen inkl. Leerzeichen, kommen Tabellen oder Abb. hinzu entsprechend weniger.

Diagnosekriterien im schnellen Überblick

Hypertonie:

Systolischer Blutdruck $>140 \mathrm{mmHg}$ Diastolischer Blutdruck $>90 \mathrm{mmHg}$

(in Ruhe innerhalb von $6 \mathrm{~h}$ zweimal gemessen)
Präeklampsie: Hypertonie Proteinurie $(\geq 3 \mathrm{~g} / 24 \mathrm{~h})$
Eklampsie: Tonischklonische Krampfanfälle

\section{HELLP-Syndrom:}
H Hemolysis
Hämolyse: LDH $\uparrow$ Haptoglobin $\downarrow$
EL Elevated Liver Enzyms
LP Low Platelets
Erhöhte Leberenzyme: GOT, GPT $\uparrow$ Erniedrigte Thrombozyten $<100 \mathrm{G} / \mathrm{l}$ 


\section{Ursachen einer Hypertonie}

Die HTN ist eine multifaktorielle, polygene Erkrankung, in der unter anderem Genetik, Ernährung, Stressfaktoren, Rauchen, zunehmendes Alter sowie fehlende Bewegung eine Rolle spielen.

\section{Ursachen der Präeklampsie und deren Folgen}

Nach derzeitigem Stand der Wissenschaft sind die Ursachen der (Prä-)Eklampsie und des HELLP-Syndrom nicht abschliessend geklärt. Vermutet wird eine Implantationsstörung des Trophoblasten in die Uteruswand, die zu einer Minderperfusion der Plazenta führt. Ursächlich wird die Freisetzung toxischer Stoffe und periphere Vasokonstriktion angenommen, die in einer HTN der Mutter sowie in Endothelschäden und Bildung von Mikrothromben resultieren. Als Komplikation kann es zu einer generalisierten Mikrozirkulationsstörung kommen, die zu einer Schädigung der Endorgane führt [18].

\section{Differentialdiagnose der primären/idiopathischen Hypertonie}

Bei jeder Erstdiagnose einer HTN sollte differentialdiagnostisch bei folgenden Konstellationen an eine sekundäre Hypertonie gedacht werden:

HTN in der Jugend ( $<30$. Lebensjahr, besonders bei negativer Familienanamnese für HTN), sehr hohe BD-Werte, plötzlich aufgetretene HTN oder plötzliche Verschlechterung einer vorbestehenden HTN, plötzlich neu aufgetretene Niereninsuffizienz oder Verschlechterung einer Niereninsuffizienz (besonders nach Gabe eines ACE-Hemmers oder AT2-Blockers), ausgeprägte Atherosklerose und multiple Herz-KreislaufRisikofaktoren [19].

\section{(1517)}

\section{Therapie, Prognose und Prävention}

Maximal 3000 Zeichen inkl. Leerzeichen, kommen Tabellen oder Abb. hinzu entsprechend weniger.

\section{Therapie}

\section{Medikamentöse antihypertensive Therapie}

Der Beginn einer medikamentösen BD-senkenden Therapie in der Schwangerschaft sollte immer kritisch hinterfragt werden und wird deshalb bei unkompliziertem Verlauf erst bei BD-Werten über 160/110 mmHg empfohlen. Diese sollte aber auch bei BD-Werten von 140/90 mmHg bei schwangerschaftsabhängiger HTN, subklinischen Organschäden oder Symptomen sowie persistierendem BD über 150/95 mmHg erwogen werden [3]. 
Tabelle 4: Langzeitbehandlung mit oralen Hypertensiva $[1,20,21]$

\begin{tabular}{|c|c|c|c|}
\hline & Medikament & Dosierung & Anmerkung \\
\hline 1. Wahl & $\begin{array}{l}\text { Alpha-Methyldopa } \\
\text { Labetalol }\end{array}$ & $\begin{array}{l}250-500 \mathrm{mg} \text { oral, } \\
(2-4 \mathrm{x} / \mathrm{d}), \mathrm{max} .2 \mathrm{~g} / \mathrm{d} \\
100-1200 \mathrm{mg} \text { oral, } \\
(2 \mathrm{x} / \mathrm{d}) \\
50 \mathrm{mg} \text { langsam iv., } \\
\text { max. } 200 \mathrm{mg}\end{array}$ & $\begin{array}{l}\text { eingeschränkter Gebrauch bei } \\
\text { Depression } \\
\text { Akuttherapie bei hypertensiven } \\
\text { Notfällen }\end{array}$ \\
\hline 2. Wahl & Nifedipin retard & $\begin{array}{l}20-60 \mathrm{mg} \text { oral, } \\
\max .120 \mathrm{mg} / \mathrm{d}\end{array}$ & nicht im 1. Trimenon \\
\hline 2. Wahl & $\begin{array}{l}\text { Selektive } \beta \text {-1-Blocker, } \\
\text { Metoprolol als } \\
\text { Mittel der Wahl }\end{array}$ & $25-100 \mathrm{mg},(2 \mathrm{x} / \mathrm{d})$ & $\begin{array}{l}\text { Risiko fetaler Wachstums- } \\
\text { restriktion }\end{array}$ \\
\hline $\begin{array}{l}\text { NICHT } \\
\text { GEEIGNET }\end{array}$ & Diuretika & & $\begin{array}{l}\text { Beeinträchtigung der utero- } \\
\text { plazentaren Perfusion durch } \\
\text { Plasmavolumenreduktion }\end{array}$ \\
\hline $\begin{array}{l}\text { NICHT } \\
\text { GEEIGNET }\end{array}$ & ACE-Hemmer & & $\begin{array}{l}\text { teratogen, fetotoxisch, } \\
\text { nephrotoxisch für Neu- } \\
\text { geborene, Oligohydramnion }\end{array}$ \\
\hline $\begin{array}{l}\text { NICHT } \\
\text { GEEIGNET }\end{array}$ & $\begin{array}{l}\text { Angiotensin, } \\
\text { AT1-Antagonisten }\end{array}$ & & $\begin{array}{l}\text { Oligohydramnion, } \\
\text { Schädelknochenhypoplasie } \\
\text { teratogen und nephrotoxisch }\end{array}$ \\
\hline $\begin{array}{l}\text { NICHT } \\
\text { GEEIGNET }\end{array}$ & $\begin{array}{l}\text { Alle anderen } \\
\text { Antihypertensiva }\end{array}$ & & $\begin{array}{l}\text { ungenügende Informationen } \\
\text { vorliegend }\end{array}$ \\
\hline
\end{tabular}

Entscheidet man sich für eine medikamentöse Therapie werden BD-Werte zwischen 130/80 und 150/100 $\mathrm{mmHg}$ angestrebt [21].

\section{Lifestyle-Änderung in der Schwangerschaft}

Spezielle Kost und Bettruhe zur Vermeidung von hypertonen Krisen sollten nur nach Abwägung des Nutzen-Risikoprofils und nicht routinemässig empfohlen werden. Eine Restriktion in der Kochsalzaufnahme kann sogar negative Effekte erzielen, da diese wie 
Diuretika zur Reduktion des Plasmavolumens und somit zur Abnahme der

uteroplazentaren Perfusion führen kann. Die Vermeidung von psychischer und physischer Belastung und häufige Ruhepausen wirken supportiv. Eine Arbeitsunfähigkeitsbescheingung sollte in Erwägung gezogen werden [9, 22]. (579)

\section{Überwachung der fetalen Entwicklung}

Bei Frauen mit 1. und 2. gradiger HTN sollte mit einer Ultraschalluntersuchung

(Fetometrie, Messung der Fruchtwassermenge, Doppler) und CTG in der 18.-20. SSW

begonnen werden und monatlich wiederholt werden. Eine Geburtseinleitung sollte in der

38.-40. SSW angestrebt werden $[17,23,24]$. (325)

\section{Prognose}

Die meisten Patientinnen mit Präeklampsie zeigen nach der Entbindung eine sofortige BD-Normalisierung. Zu beachten ist, dass eine Präeklampsie auch pp auftreten kann (meist in den ersten 48h). Vor dem Abschluss der Behandlung sollten die Patientinnen auf das hohe Wiederholungsrisiko dieser Erkrankungen in späteren Schwangerschaften aufmerksam gemacht werden (25\% aller Patientinnen mit Präeklampsie und $13 \%$ mit HELLP) [1].

\section{Prävention}

HTN in der Schwangerschaft ist ein bedeutsamer und eigenständiger Risikofaktor für das Auftreten kardiovaskulärer Erkrankungen im späteren Leben. Diesem Patientenkollektiv wird daher eine frühzeitige kardiovaskuläre Prävention (ab dem 40. Lebensjahr) mit lebenslangen Verlaufskontrollen empfohlen [25, 26].

Bei Frauen mit Risikofaktoren hat sich eine orale Acetylsalicylat-Dosierung von $100 \mathrm{mg} / \mathrm{d}$ ab der Früh-Schwangerschaft (bis spätestens 16. SSW) bis zur 34+0 SSW etabliert. Eine generelle Acetylsalicylat-Prophylaxe ist jedoch nicht indiziert [27, 28]. (3357) 


\section{Fallbericht chronische Hypertonie in der Schwangerschaft}

\section{U} HTN und positiver Familienanamnese bezüglich Präeklampsie stellt sich in der Praxis zur Ausstellung eines neuen Medikamentenrezeptes vor. Im Gespräch erzählt Sie Ihnen von Ihrem aktuell bestehenden Kinderwunsch. Sie habe deshalb ihre hormonelle Kontrazeption selbstständig abgesetzt und nehme seither als einzige Medikation einen ACE-Hemmer.
Weg zur Diagnose/Kommentar

Aufgrund des Alters, der chronischen schwangerschaftsunabhängigen HTN, sowie positiver Familienanamnese für Präeklampsie, handelt es sich um eine potentielle Risiko-Schwangerschaft. ACE-Hemmer haben eine teratogene sowie fetotoxische Wirkung. Daher sollten Frauen im gebärfähigen Alter ACEHemmer nur in Kombination mit einem Kontrazeptivum einnehmen.

Weg zur Diagnose/Kommentar

$>35$. Lebensjahr $=$ Risikoschwangerschaft normalgewichtige Patientin chronische HTN, aktuell unter medikamentöser Therapie normotensive BD-Werte, unauffälliger internistischer Status
1. $125 / 82 \mathrm{mmHg}$

Puls $86 / \mathrm{min}$, unauffällige kardiopulmonale Auskultation, keine Herzgeräusche, keine Halsvenenstauung, keine Beinödeme Abdomen weich, keine Druckdolenzen, keine Organomegalien tastbar.

\section{Aktuelle Therapie \\ Lisinopril $5 \mathrm{mg} / \mathrm{Tag}$}

\section{Routinelabor}

$\mathrm{Na}$

$\mathrm{K}$

Kreatinin

eGFR (CKD)

AST

ALT

TSH

Glucose nüchtern $4.3 \mathrm{mmol} / \mathrm{L}$

$\mathrm{Hb}$

$129 \mathrm{~g} / \mathrm{L}$

Erythrozyten $\quad 4.28 \mathrm{~T} / \mathrm{L}$

Leukozyten $7.41 \mathrm{G} / \mathrm{L}$

Thrombozyten 172 G/L

\section{Spontanurin}

Glucose

Protein

$\mathrm{pH}$

$\begin{array}{ll}\text { negativ } & \text { qualitativ } \\ \text { negativ } & \text { qualitativ } \\ 5.5 & (5.0-7.5)\end{array}$

$(136-145)$

(3.3-4.5)

$(44-80)$

(>90)

$(<35)$

(10-35)

(0.16-4.25)

$(<5.6)$

$(117-153)$

(3.0-9.6)

(143-400)

\section{Cave: ACE-Hemmer gelten als potentiell} teratogen und fetotoxisch.

Kein Hinweis für eine Nierenerkrankung oder Diabetes mellitus als mögliche Risikofaktoren für eine Präeklampsie. Bei Schwangerschaftswunsch zusätzlich Tyreothropin (TSH) bestimmt.

Normwertige Transaminasen und Thrombozytenwerte, unauffälliger Blutstatus.

Keine Glucosurie, keine Proteinurie, Normwertiger Albumin/Kreatinin-Quotient im Spontanurin. 


\begin{tabular}{|c|c|}
\hline $\begin{array}{lll}\text { Dichte } & 1.021 \mathrm{~g} / \mathrm{mL} & (1.020-1.030) \\
\text { Leukozyten } & \text { negativ } & \text { qualitativ } \\
\text { Plattenepithel } & \text { negativ } & \text { qualitativ } \\
\text { Protein } & 0.065 \mathrm{~g} / \mathrm{L} & (<0.15) \\
\text { Albumin } & 5.3 \mathrm{mg} / \mathrm{L} & \\
\text { Albumin/Kreatinin } & 0.7 \mathrm{mg} / \mathrm{mmol}(<2.26) \\
\text { Schwangerschaftstest negativ } & \\
\end{array}$ & Keine Schwangerschaft. \\
\hline $\begin{array}{l}\text { サ Therapie der Patientin } \\
\text { Neben allgemeinen Informationen zur Schwanger } \\
\text { allem über die potentiell teratogene, fetotoxische } \\
\text { Schwangerschaftswunsch eine Umstellung auf Al } \\
\text { Sowohl familienanamnestisch als auch aufgrund } \\
\text { chronischen HTN handelt es sich bei der geplante } \\
\text { Schwangerschaft. Erfreulicherweise aktuell unauf } \\
\text { Labor. } \\
\text { Die Patientin wird ausführlich über Warnsymptom } \\
\text { zeitnahe Verlaufskontrolle nach Umstellung der a } \\
\text { Regelmässige Heim-BD-Messungen mit einem fü } \\
\text { sowie das Führen eines BD-Tagebuches werden }\end{array}$ & $\begin{array}{l}\text { schaft, informieren wir die Patientin vor } \\
\text { Nirkung von ACE-Hemmern und führen bei } \\
\text { ha-Methyldopa durch. } \\
\text { les Alters und der vorbestehenden } \\
\text { n Schwangerschaft um eine Risiko- } \\
\text { älliger klinischer Status und normwertiges } \\
\\
\text { einer Präeklampsie informiert und eine } \\
\text { tihypertensiven Therapie vereinbart. } \\
\text { Schwangere validierten BD-Messgerät } \\
\text { eschlossen. }\end{array}$ \\
\hline
\end{tabular}


Fragen zur chronischen Hypertonie in der Schwangerschaft

\author{
Hinweise zum Verfassen der MC-Fragen \\ 1. Fragetypen: Verwenden Sie nur Typ-A-Fragen (Einfachauswahl: 5 \\ Antwortmöglichkeiten, nur eine ist richtig.) \\ und \\ (Mehrfachauswahl: 5 Antwortmöglichkeiten, mehrere sind richtig. \\ 2. Achten Sie darauf, dass die Fragen klar und eindeutig sind. \\ 3. Keine Fangfragen! \\ 4. Es soll nur das im Artikel vermittelte Wissen abgefragt werden.
}

\title{
Frage 1
}

Welche Aussagen zur chronischen Hypertonie in der Schwangerschaft stimmen? (Mehrfachauswahl)

a) Bei Frauen mit chronischer Hypertonie beobachtet man während der Schwangerschaft ein signifikant erhöhtes Risiko von Plazentalösung, fetaler Wachstumsrestriktion und Frühgeburtlichkeit.

b) Die Ursachen eines Hypertonus werden meist als multifaktoriell zusammengefasst.

c) Bei einer chronischen Hypertonikerin kann im ersten Trimester der Schwangerschaft ein Abfall der mittleren Blutdruckwerte ohne Medikation beobachtet werden.

d) First-Line Therapie zur Senkung der Hypertonie in der Schwangerschaft ist die Restriktion in der täglichen Kochsalzaufnahme.

e) Alpha-Methyldopa, Calcium-Antagonisten und Betablocker können in der Behandlung einer chronischen Hypertonie in der Schwangerschaft eingesetzt werden.

\section{Frage 2}

In der Schwangerschaft ist die Messung des Blutdrucks wichtig, da eine Blutdruckerhöhung auf eine Präeklampsie hinweisen kann und somit eine uteroplazentare Funktionsstörung frühzeitig erkannt werden kann.

Welche der folgenden Untersuchungen wird bei Verdacht einer Dysfunktion der Plazenta eingesetzt? (Einfachauswahl)
a) Aufzeichnung fetaler Atembewegungen
b) Amnioskopie zum Nachweis mekoniumhaltigen Fruchtwassers
c) Vaginaler Ultraschall 
d) Amniozentese zur Bestimmung der Bilirubinoid-Konzentration im Fruchtwasser mittels Spektrophotometrie ( $\triangle \mathrm{OD} 450 \mathrm{~nm}$ )

e) Sonographie der A. umbilicalis und der fetalen A. cerebri media

\section{Frage 3}

Eine ihnen lange bekannte Patienten mit chronischer Hypertonie erscheint zum Termin und berichtet Ihnen im Gespräch, dass sie im 2. Monat schwanger sei. Welches dieser folgenden Ergebnisse der notwendigen Anamnese und Diagnostik alarmiert Sie? (Mehrfachauswahl)

a) Blutdruckwert 130/80 mmHg nach einmaliger Messung im Sitzen mit Hilfe einer adäquaten Blutdruckmanschette

b) Keine Spur Eiweiss im Urin-Schnelltest

c) Aktuelle Medikation mit L-Thyroxin 75 ug und Lisinopril $2.5 \mathrm{mg}$

d) Nachweis von $150 \mathrm{mg}$ Protein im 24h-Sammelurin

e) nächtliche Hypertonien in der 24h-Blutdruckmessung

\section{Frage 4}

Eine 30-Jährige stellt sich in der 32. SSW vor und beklagt Schmerzen im rechten Oberbauch. Der von Ihnen gemessene Blutdruck der Patientin liegt bei 160/95 mmHg. Sie erkennen die möglichen Gefahren und überweisen die Patientin sofort in ein Krankenhaus.

Welche der Laboruntersuchungen ist zur weiteren Diagnostik hinsichtlich der hier am ehesten in Betracht zu ziehenden Erkrankung vordringlich dort zu veranlassen? Bestimmung der Konzentration von? (Mehrfachauswahl)

a) Alpha-Fetoprotein, CDT (Carbohydrate Deficient Transferrin)

Formatiert: Französisch (Schweiz)

b) LDH, Bilirubin, Haptoglobin

c) Thrombozyten

d) GPT (ALT), GOT (AST)

e) CRP, Blutsenkung

\section{Frage 5}

Eine 45-jährige Patientin stellt sich zum Check-up in Ihrer Praxis vor. Im Gespräch berichtet sie, sie fühle sich gesund und nehme keine regelmässigen Medikamente ein. Bei der persönlichen Anamnese erfahren Sie, dass man während der 
Schwangerschaft ihres einzigen Kindes einen erhöhten Blutdruck und Eiweisse im Urin festgestellt hatte. Nach der Entbindung sei so etwas nicht mehr aufgetreten. Zum Check-up welchen Organsystems raten Sie dieser Patientin aufgrund der Anamnese jährlich? (Einfachauswahl)

Check-up des
a) neurologischen System
b) endokrinologischen System
c) kardiovaskulären System
d) hepatologischen System
e) nephrologischen System 


\section{Antworten zu den Fragen zur Hypertonie in der Schwangerschaft aus PRAXIS Nr.}

Frage 1

Richtig ist/sind Antwort/en a), b), c), e)

Kommentar zur / zu den richtigen Antworten:

Ad a) Chronische Hypertonie kann zu einer gestörten Durchblutung der Plazenta führen, welche u.a. Plazentalösung, vermindertes Wachstum des Feten und Frühgeburtlichkeit zur Folge haben kann.

Ad b) Die Ursachen eines Hypertonus sind multifaktorieller, polygener Genese. Genetik, Ernährungsfaktoren, Stressfaktoren, Rauchen, zunehmendes Alter sowie fehlende Bewegung können hierbei eine Rolle spielen.

Ad c) Im Laufe jeder Schwangerschaft sinkt der mittlere Blutdruckwert durch eine erhöhte Ausschüttung von Progesteron im ersten Trimester. Im letzten Drittel der Schwangerschaft erreicht der Blutdruck in der Regel präkonzeptionell gemessene Blutdruckwerte. Diese physiologische Veränderung in der Schwangerschaft erfordert regelmässige Kontrollen des Blutdrucks und Evaluation einer bestehenden antihypertensiven Therapie.

Ad e) Der Beginn einer antihypertensiven Therapie mit Alpha-Methyldopa, CalciumAntagonisten (v.a. Nifedipin) oder selektiven Betablocker (v.a. Metoprolol) kann in der Schwangerschaft nach sorgfältiger Abwägung von Nutzen und Risiko vertreten werden.

\section{Die Aussage d) ist falsch:}

Kommentar zu den falschen Antworten:

Ad d) Von Restriktion in der täglichen Kochsalzaufnahme zur Behandlung einer Hypertonie in der Schwangerschaft sollte abgeraten werden. Eine verminderte Kochsalzaufnahme kann zu einer Reduktion des Plasmavolumens und somit zur Abnahme der uteroplazentaren Perfusion führen.

\section{Frage 2}

\section{Richtig ist/sind Antwort/en e)}

Kommentar zur / zu den richtigen Antworten:

Eine nicht-invasive und genaue Methode zur intensivierten fetalen Überwachung ist die Doppler-Sonographie fetaler und maternaler Gefässgebiete (A. umbilicalis, A. uterina, fetaler A. cerebri media). Der Blutfluss in diesen Gefässen korreliert mit der uteroplazentaren Funktion und dem Präeklampsierisiko. 
Falsch sind Antwort a), b), c), d)

Kommentar zu den falschen Antworten:

$\mathrm{Ad}$ a): Fetale Atembewegungen sind während der Schwangerschaft physiologisch und nicht mit Schwangerschaftshypertonie oder Präeklampsie assoziiert.

Ad b): Bei der Amnioskopie wird das Fruchtwasser mit einem durch den geöffneten Muttermund eingeführten Endoskop auf Mekoniumabgang untersucht. Nachweis von Mekonium kann nur einen generellen Hinweis auf ein erhöhtes Stresslevel des Feten geben.

Ad c): Die Dysfunktion der Plazenta wird mit Hilfe einer abdominalen Doppler-

Sonographie bestimmt.

Ad d): Die invasive Messung der Bilirubinoide im Fruchtwasser durch Punktion des Amnion durch die Bauchhaut der Mutter dient dem Nachweis von irregulären mütterlichen Autoantikörpern (z.B. bei Rhesus-Inkompatibilität) und korreliert nicht mit dem Vorliegen einer Präeklampsie.

\section{Frage 3}

\section{Richtig ist/sind Antwort/en c), e)}

Kommentar zur / zu den richtigen Antworten:

Von den genannten Möglichkeiten ist die aktuelle Medikation mit einem ACE-Hemmer alarmierend, da ACE-Hemmer in der Schwangerschaft u.a. als fetotoxisch gelten. ACEHemmer müssen am besten vor Beginn einer Schwangerschaft durch geeignete Antihypertensiva (siehe Frage 2) ersetzt werden. Ausserdem können die nächtlichen Episoden überhöhten Blutdrucks auf eine Umkehr des zirkadianen Blutdruckrhythmus in der Schwangerschaft hinweisen. Untersuchungen haben gezeigt, dass diese Veränderung im Zusammenhang mit dem Auftreten einer Präeklampsie steht.

\section{Falsch sind Antworten a), b), d)}

Kommentar zu den falschen Antworten:

Ad a) Ein einmaliger Blutdruckwert von 130/80 mmHg gilt per definitionem nicht als Bluthochdruck ( $\geq 140 / 90 \mathrm{mmHg}$ ). Regelmässige Überwachung des Blutdruckes ist jedoch besonders in der Schwangerschaft anzuraten. Ausserdem gilt es zu bedenken, dass erst eine wiederholt erhöhte Blutdruckmessung in Abstand von mindestens 4-6 Stunden in entspannter Atmosphäre einen Hinweis auf das Vorliegen einer Hypertonie geben kann. Ad b), d) Die Resultate im Screening Test und des 24h Sammelurins stellen Normalbefunde dar. 


\section{Frage 4}

Richtig ist/sind Antwort/en b), c), d)

Kommentar zur / zu den richtigen Antworten:

Ein Blutdruck von 160/95 mmHg bei dieser Schwangeren im dritten Trimenon stellt einen Hinweis für das Vorliegen einer Präeklampsie dar. Der durch eine erhöhte Leberkapselspannung ausgelöste rechtsseitige Oberbauchschmerz ist zudem ein Warnzeichen für eine gefürchtete Komplikation dieser Erkrankung: das HELLP-Syndrom ( $\mathrm{H}=$ Hemolysis, $\mathrm{EL}=$ Elevated Liver Enzyme Levels, $\mathrm{LP}=$ Low Platelet Count). Neben Hämolysezeichen (LDH, Bilirubin, Haptoglobin) und einer Erhöhung der Transaminasen (GPT, GOT) ist ein erniedrigter Thrombozytenwert diagnostisch entscheidend. Bei einer schwangeren Frau mit kritisch erhöhtem Blutdruck gehören weiter genannte Marker nicht zur vordringlichen Diagnostik.

\section{Falsch sind Antworten a), e)}

Kommentar zu den falschen Antworten:

Die Konzentration von Alpha-Fetoprotein, freiem Estriol und der freien beta-Kette des Choriongonadotropins wird in der Pränataldiagnostik beim sogenannten Triple-Test im Blut der Mutter untersucht. Dieser Suchtest kann Mütter identifizieren, die eine erhöhte Wahrscheinlichkeit haben, ein Kind mit chromosomaler Besonderheit (z.B. Trisomie 21) oder eine Neuralfehlbildung auszutragen. Um den Verdacht zu bestätigen, müssen dann meist invasive Methoden folgen.

Als Carbohydrate Deficient Transferrin (CDT) wird die Summe der veränderteren Strukturen des Transferrins benannt, die einen spezifischen Marker für einen chronischen Alkoholismus darstellen. Bei mindestens einwöchiger Einnahme von mehr als $60 \mathrm{~g}$ Alkohol (Ethanol) pro Tag findet sich diese typische Anreicherung von CDT und ist wochenlang im Serum nachweisbar.

Ad e) CRP und Blutsenkungsgeschwindigkeit sind sinnvolle Marker in der Entzündungsdiagnostik.

\section{Frage 5}

\section{Richtig ist Antwort c)}

Kommentar zur / zu den richtigen Antworten:

Die Präeklampsie ist mit einer erhöhten Rate von (schwangerschaftsunabhängigen) kardiovaskulären Zwischenfällen im späteren Leben assoziiert. Deshalb ist ein genaueres Augenmerk auf das kardiovaskuläre Organsystem ratsam und frühzeitige und lebenslange Prävention zu empfehlen. 
Falsch sind Antworten a), b), d), e)

Kommentar zu den falschen Antworten:

Für alle anderen Organsysteme gelten andere alters- und patientengerechten Indikationen für Check-up-Untersuchungen. 


\section{Autoren}

\section{cand. med. Tanja K. Eggersmann}

Ludwig-Maximilians-Universität München

Dr. med. univ. Stefan Zechmann

Klinik und Poliklinik für Innere Medizin, UniversitätsSpital Zürich

PD Dr. med. Lukas Zimmerli

Medizinische Klinik, Kantonsspital Olten

\section{Korrespondenzadresse}

Dr. med. univ. Stefan Zechmann

Klinik und Poliklinik für Innere Medizin

Universitätsspital

Rämistrasse 100

8091 Zürich

Stefan.zechmann@usz.ch

\section{Bibliographie}

3 bis 5 für den Praktiker relevante und leicht zugängliche Literaturhinweise.

AWMF, 015/018 RN. S1-Leitlinie: Diagnostik und Therapie hypertensiver Schwangerschaftserkrankungen 2013.

Mancia, G., Fagard, R., et. al. 2013 ESH/ESC Guidelines for the management of arterial hypertension. J Hypertens 2013; 31: 1281-1357.

Report of the National High Blood Pressure Education Program Working Group on High Blood Pressure in Pregnancy. Am J Obstet Gynecol 2000; 183: S1-S22.

Seely EW, Ecker J. Chronic hypertension in pregnancy. Circulation. 2014; 129(11): 1254-61.

Suter P, Sudano I. Wann und wie soll man eine sekundäre Ätiologie abklären? Schweiz Med Forum 2014;14(08):146-150. 
1. AWMF, 015/018 RN. S1-Leitlinie: Diagnostik und Therapie hypertensiver Schwangerschaftserkrankungen 2013.

2. Seely EW, Ecker J. Chronic hypertension in pregnancy. Circulation. 2014; 129(11): 1254-61.

3. Mancia G, Fagard R, Narkiewicz K, Redon J, Zanchetti A, Bohm M, et al. 2013 ESH/ESC

Guidelines for the management of arterial hypertension: the Task Force for the management of arterial hypertension of the European Society of Hypertension (ESH) and of the European Society of Cardiology (ESC). Journal of hypertension. 2013; 31(7): 1281-357.

4. von Dadelszen P, Ornstein MP, Bull SB, Logan AG, Koren G, Magee LA. Fall in mean arterial pressure and fetal growth restriction in pregnancy hypertension: a meta-analysis. Lancet. 2000; 355(9198): 87-92.

5. Abalos E, Duley L, Steyn DW. Antihypertensive drug therapy for mild to moderate hypertension during pregnancy. The Cochrane database of systematic reviews. 2014; 2: Cd002252.

6. Bodnar LM, Ness RB, Markovic N, Roberts JM. The risk of preeclampsia rises with increasing prepregnancy body mass index. Annals of epidemiology. 2005; 15(7): 475-82.

7. ACOG Committee Opinion number 315, September 2005. Obesity in pregnancy. Obstetrics and gynecology. 2005; 106(3): 671-5.

8. Sibai BM, Lindheimer M, Hauth J, Caritis S, VanDorsten P, Klebanoff M, et al. Risk factors for preeclampsia, abruptio placentae, and adverse neonatal outcomes among women with chronic hypertension. National Institute of Child Health and Human Development Network of Maternal-Fetal Medicine Units. The New England journal of medicine. 1998; 339(10): 667-71.

9. Homuth V, Derer W, Dechend R. [Hypertension and pregnancy]. Deutsche medizinische Wochenschrift (1946). 2009; 134(5): 195-200.

10. Higgins JR, de Swiet M. Blood-pressure measurement and classification in pregnancy. Lancet. 2001; 357(9250): 131-5.

11. Brown MA, Buddle ML, Farrell T, Davis G, Jones M. Randomised trial of management of hypertensive pregnancies by Korotkoff phase IV or phase V. Lancet. 1998; 352(9130): 777-81.

12. Brown MA, Lindheimer MD, de Swiet M, Van Assche A, Moutquin JM. The classification and diagnosis of the hypertensive disorders of pregnancy: statement from the International Society for the Study of Hypertension in Pregnancy (ISSHP). Hypertension in pregnancy. 2001; 20(1): Ix-xiv.

13. O'Brien E, Parati G, Stergiou G, Asmar R, Beilin L, Bilo G, et al. European Society of Hypertension position paper on ambulatory blood pressure monitoring. Journal of hypertension. 2013; 31(9): 1731-68.

14. Rojo-Contreras W, Salazar-Paramo M, Parra-Carrillo JZ, Ilnigo-Riesgo CA, Bonilla-Rosales I, Villa-Manzano AI, et al. [Circadian rhythm of blood pressure in patients with chronic hypertension and pregnancy]. Ginecologia y obstetricia de Mexico. 2014; 82(9): 604-12.

15. Wilson M, Morganti AA, Zervoudakis I, Letcher RL, Romney BM, Von Oeyon P, et al. Blood pressure, the renin-aldosterone system and sex steroids throughout normal pregnancy. The American journal of medicine. 1980; 68(1): 97-104

16. August P, Lenz T, Ales KL, Druzin ML, Edersheim TG, Hutson JM, et al. Longitudinal study of the renin-angiotensin-aldosterone system in hypertensive pregnant women: deviations related to the development of superimposed preeclampsia. American journal of obstetrics and gynecology. 1990; 163(5 Pt 1): 1612-21.

17. Report of the National High Blood Pressure Education Program Working Group on High Blood

Pressure in Pregnancy. American journal of obstetrics and gynecology. 2000; 183(1): S1-s22.

18. Chaiworapongsa T, Chaemsaithong P, Yeo L, Romero R. Pre-eclampsia part 1: current understanding of its pathophysiology. Nature reviews Nephrology. 2014; 10(8): 466-80.

19. Suter P, Sudano I. Hypertonie: Wann und wie soll man eine sekundäre Ätiologie abklären? Schweiz Med Forum 2014; 14(08):146-150.

20. Magee LA, Abalos E, von Dadelszen P, Sibai B, Easterling T, Walkinshaw S. How to manage hypertension in pregnancy effectively. British journal of clinical pharmacology. 2011; 72(3): 394-401.

21. Seely EW, Ecker J. Clinical practice. Chronic hypertension in pregnancy. The New England journal of medicine. 2011; 365(5): 439-46.

22. Meher S, Abalos E, Carroli G. Bed rest with or without hospitalisation for hypertension during pregnancy. The Cochrane database of systematic reviews. 2005(4): Cd003514.

23. ACOG Practice Bulletin No. 125: Chronic hypertension in pregnancy. Obstetrics and gynecology. 2012; 119 (2 Pt 1): 396-407.

24. Sibai BM. Chronic hypertension in pregnancy. Obstetrics and gynecology. 2002; 100(2): $369-77$.

25. Harskamp RE, Zeeman GG. Preeclampsia: at risk for remote cardiovascular disease. The American journal of the medical sciences. 2007; 334(4): 291-5.

26. Luft FC. Pre-eclampsia and the maternal cardiovascular risk. Nephrology, dialysis, transplantation : official publication of the European Dialysis and Transplant Association - European Renal Association. 2003; 18(5): 860-1.

27. Askie LM, Duley L, Henderson-Smart DJ, Stewart LA. Antiplatelet agents for prevention of preeclampsia: a meta-analysis of individual patient data. Lancet. 2007; 369(9575): 1791-8. 
28. Ayala DE, Ucieda R, Hermida RC. Chronotherapy with low-dose aspirin for prevention of complications in pregnancy. Chronobiology international. 2013; 30(1-2): 260-79. 